\title{
Extended-spectrum $\beta$-lactamase-producing Klebsiella pneumoniae from blood culture
}

Bloodstream infections (BSIs) caused by extended-spectrum $\beta$-lactamase-producing Klebsiella pneumoniae (ESBL-KP) isolates are a major concern for clinicians, since they markedly increase the rates of treatment failure and death particularly in intensive care units and amongst paediatric patients and also in medical and surgical wards. In recent years, following extensive use of the expanded-spectrum cephalosporins, outbreaks of infection caused by ESBL-KP have been widely reported throughout the world (Branger et al., 1998). ESBLs are a rapidly evolving group of $\beta$-lactamases which share the ability to hydrolyse third-generation cephalosporins and aztreonam, yet are inhibited by clavulanic acid (Paterson \& Bonomo, 2005). Unfortunately, ESBL-producing organisms often also possess resistance determinants to other important antibiotic groups, such as aminoglycosides and fluoroquinolones, leaving an extremely limited range of effective agents. Carbapenems play an important role in the treatment of infections caused by ESBL-KP. The $\beta$-lactamase-mediated carbapenem resistance among K. pneumoniae isolates and others is an emerging problem (Tenover et al., 2006). We conducted a microbiological study to assess the prevalence of ESBL-KP, to determine its resistance pattern with special reference to meropenem susceptibility and its contribution to mortality, in our tertiary care hospital.

Blood cultures submitted between January 2006 and July 2006 were examined. Repeat samples were excluded. A biphasic medium consisting of brain heart infusion (BHI) agar and BHI broth with sodium polyanethol sulphonate as an anticoagulant was used for blood cultures. K. pneumoniae was identified according to routine bacteriological procedures.

Susceptibility testing was performed by the disc diffusion method and isolates were screened for the ESBL phenotype. The tests were performed according to CLSI (formerly NCCLS) guidelines (CLSI, 2005). An ESBL confirmatory disc diffusion assay was done by the Oxoid combination disk method (Carter et al., 2000). It depends on the comparison of the inhibition zones given by cefpodoxime $(10 \mu \mathrm{g})$ and cefpodoxime-plus-clavulanate $(10 \mu \mathrm{g}$ plus $1 \mu \mathrm{g})$ discs. $\mathrm{A} \geqslant 5 \mathrm{~mm}$ increase in the zone diameter in the presence of the clavulanic acid disc versus the cephalosporin disc alone was interpreted as phenotypic evidence of ESBL production.
During the study, 130 patients with $K$. pneumoniae BSI isolates were identified. Of the 130 patients, $119(91.5 \%)$ were from the neonatal (NICU) and paediatric (PICU) intensive care units and only 11 ( $8.5 \%)$ were adult patients. Of the 130 isolates, a very high proportion, 126, produced ESBLs (97\%) (Table 1). This is one of the highest prevalence rates of ESBL infection described. Of the 118 ESBL-KP NICU and PICU isolates, 18 (15.3\%) were meropenem-resistant and all of the patients from whom these were isolated died. Of the 11 isolates from adults, eight were ESBL-KP, and of these two $(25 \%)$ were meropenem-resistant. These two patients deteriorated and left the hospital against medical advice. Of all the ESBL-KP isolates, about half ( $49 \%)$ of them were susceptible to amikacin.

BSIs caused by ESBL-producing strains of $K$. pneumoniae represent a serious clinical problem associated with a high mortality rate particularly in intensive care units and amongst paediatric patients. The $\beta$-lactamase-mediated carbapenem resistance among K. pneumoniae isolates and others is an emerging problem. The ability of K. pneumoniae to spread rapidly amongst patients often leads to outbreaks of nosocomial infection, especially in

Table 1. In vitro antimicrobial susceptibilities of $130 \mathrm{~K}$. pneumoniae isolates

\begin{tabular}{|c|c|c|}
\hline Antimicrobial agent & $\begin{array}{c}\text { Susceptible no. }(\%) \text { of } \\
\text { non-ESBL-producing } \\
\text { K. pneumoniae }(n=4 ; 3 \%)\end{array}$ & $\begin{array}{c}\text { Susceptible no. }(\%) \text { of } \\
\text { ESBL-producing } \\
\text { K. pneumoniae }(n=126 ; 97 \%)\end{array}$ \\
\hline Ampicillin $(10 \mu \mathrm{g})$ & $0(0)$ & $0(0)$ \\
\hline Amikacin $(30 \mu \mathrm{g})$ & $2(50)$ & $62(49)$ \\
\hline Gentamicin $(10 \mu \mathrm{g})$ & $0(0)$ & $0(0)$ \\
\hline Chloramphenicol $(30 \mu \mathrm{g})$ & $0(0)$ & $12(10)$ \\
\hline Ciprofloxacin $(5 \mu \mathrm{g})$ & $1(25)$ & $1(1)$ \\
\hline Ceftriaxone $(30 \mu \mathrm{g})$ & $1(25)$ & $0(0)$ \\
\hline Ceftazidime $(30 \mu \mathrm{g})$ & $1(25)$ & $0(0)$ \\
\hline Meropenem $(10 \mu \mathrm{g})$ & $4(100)$ & $107(85)$ \\
\hline
\end{tabular}

${ }^{*}$ All ESBL-producing isolates were considered resistant to penicillins, monobactam and oxyiminocephalosporins, according to CLSI guidelines. 
neonatal units. There is a need for continuous monitoring of susceptibility to carbapenems and of carbapenemase production. Our findings emphasize the urgent need for efforts for the prevention of the spread of ESBL-producing bacteria. Interventions geared towards improving methods for early identification of ESBL-producing bacteria, identifying patient populations at high risk for infection with ESBL producers and, finally, improving treatment strategies and expanding therapeutic options are greatly needed.

\section{B. N. Harish, G. A. Menezes,} S. Shekatkar and S. C. Parija
Dept of Microbiology, Jawaharlal Institute of Postgraduate Medical Education and Research (JIPMER), Pondicherry 605006, India

Correspondence: B. N. Harish (drbnharish@yahoo.com)

Branger, C., Lesimple, A. L., Bruneau, B., Berry, P. \& Lambert-Zechovsky, N. (1998). Long-term investigation of the clonal dissemination of Klebsiella pneumoniae isolates producing extended-spectrum $\beta$-lactamases in a university hospital. J Med Microbiol 47, 201-209.

Carter, M. W., Oakton, K. J., Warner, M. \& Livermore, D. M. (2000). Detection of extended-spectrum $\beta$-lactamases in klebsiellae with the Oxoid combination disk method. J Clin Microbiol 38, 4228-4232.

CLSI (2005). Performance Standards for Antimicrobial Susceptibility Testing; Fifteenth Informational Supplement. M100S15. Wayne, PA: Clinical and Laboratory Standards Institute.

Paterson, D. L. \& Bonomo, R. A. (2005).

Extended-spectrum $\beta$-lactamases: a clinical update. Clin Microbiol Rev 18, 657-686.

Tenover, F. C., Kalsi, R. K., Williams, P. P., Carey, R. B., Stocker, S., Lonsway, D., Rasheed, J. K., Biddle, J. W., McGowan, J. E., Jr \& Hanna, B. (2006). Carbapenem resistance in Klebsiella pneumoniae not detected by automated susceptibility testing. Emerg Infect Dis 12, 1209-1213. 